\title{
有机添加剂调控铜互连结构电生长及 信号传输损耗的研究
}

陈苑明 ${ }^{1,2}$, 徐佳莹 ${ }^{1}$, 张灵芝 ${ }^{1}$, 卓晓煌 ${ }^{1}$, 陈昀钊 ${ }^{1}$, 王羽中 ${ }^{1}$, 何为 ${ }^{1,2^{*}}$, 王守绪 ${ }^{1}$, 张伟华 ${ }^{2}$, 苏新虹 ${ }^{2}$, 罗毓瑶 ${ }^{2}$

1. 电子科技大学材料与能源学院, 成都 610054

2. 珠海方正科技高密电子有限公司\&珠海方正科技多层电路板有限公司, 珠海 519175

*通讯作者, Email: heweiz@uestc.edu.cn

收稿日期: 2021-07-01; 接受日期: 2021-08-04; 网络版发表日期: 2021-08-18

国家自然科学基金项目(编号：51801018和61974020)、珠海市创新团队项目(编号：ZH0405190005PWC)、广东省重点领域研发计划项目(编 号：2019B090910003)、珠海市科技项目(编号：ZH01084702180040HJL)和中国科学院学部咨询评议项目(我国电子电镀基础与工业的现状和 发展)资助

摘要铜互连结构的粗糙度严重制约高频信号传输的完整性. 研究了含不同有机添加剂的镀液体系对电生长 铜层粗粘度的影响; 采用分子模拟仿真和电化学测试, 调控了有机添加剂在铜面的竞争吸附行为, 探究了引起铜 电生长差异的可能机制. 结果表明, 与仅用单一有机添加剂的镀液体系相比, 含聚二硫二丙烷磺酸钠、聚氧乙烯聚丙乙烯嵌段共聚物和健那绿的镀液体系可电生长出表面形貌平整的铜层, 且其插入损耗值为-8.9 dB@20 GHz. 因此, 采用有机添加剂调控铜互连结构电生长的低粗糙度, 降低传输损耗, 可有效保障高频信号传输完整性.

关键词高频信号, 铜互连结构, 电生长, 有机添加剂, 插入损耗

\section{1 引言}

5G通信的信号传送和处理速度在高频区，但由于 趋肤效应的影响，高频信号集中分布在互连导体结构 的表面，信号在微米级粗䊁的导体内传输易向不同方 向发生散射、反射、停顿，从而带来信号失真、干扰 等严重问题，因此高粗糙度已难以满足信号传输完整 性的要求，互连导体结构表面粗䊁度的有效调控至关 重要 $^{[1 \sim 3]}$.

电生长技术是构建铜互连图形的有效方法. 在铜 镀液中加入少量有机添加剂能够显著改善金属电生长
的状态. Chan等 ${ }^{[4]}$ 研究了高电流密度下氯离子、添加 剂及强制对流对铜面粗敉度的影响，结果表明强制对 流相比其他因素能够显著影响铜面的粗糙度，并且配 合含有 $\mathrm{Cl}^{-}$、聚乙二醇(Polyethylene glycol, PEG)与加 速剂的配方才能获得光滑的铜面. Sudibyo等 ${ }^{[5]}$ 发现磁 场对沉积层表面形貌有显著影响，铜沉积层的致密程 度随磁场的增大而增大. Rodchanarowan等 ${ }^{[6]}$ 研究了多 种添加剂对铜粗粘度的影响, 发现明胶相比其他添加 剂有更好的整平效果, 且铜面粗䊁度随着电沉积时间 的增加不会显著增加. Woo等 ${ }^{[7]}$ 通过多种电沉积环境 下的试验得出, 在含有羟乙基纤维素(Hydroxy ethyl

引用格式: Chen Y, Xu J, Zhang L, Zhuo X, Chen Y, Wang C, He W, Wang S, Zhang W, Su X, Luo Y. Investigation of organic additives to regulate growth and signal transmission loss of copper interconnection structure. Sci Sin Chim, 2021, 51: 1511-1519, doi: 10.1360/SSC-2021-0156 
cellulose)的镀液中以 $20 \mathrm{~A} / \mathrm{m}^{2}$ 的电流密度电沉积所得的 铜面粗粘度最小, 而不含有添加剂的铜沉积表面最粗 粘. Zhang等 ${ }^{[8]}$ 研究了PEG、聚二硫二丙烷磺酸钠(Bis(sodium sulfopropyl)-disulfide, SPS)、亚乙基硫脲三种 添加剂对铜面粗粘度的影响作用，研究发现：在含有 PEG的原始镀液中加入亚乙基硫脲对铜电沉积的形貌 及质量无明显改变; 而加入SPS后铜面粗粘度显著减 小, 但晶粒尺寸变化不大; 当亚乙基硫脲和SPS同时作 用时，两者存在协同作用将晶粒尺寸和铜面粗糙度降 到最低, 铜沉积的质量较好. 顾晓清等 ${ }^{[9]}$ 在硫酸铜镀液 中研究了整平剂、抑制剂、加速剂对电沉积铜层性能 的影响，结果表明整平剂在超填充中虽然没有决定性 的影响作用, 但能够避免铜晶粒的过度生长, 降低电 沉积铜层的粗䊁度.

铜电生长技术是印制电路板解决孔、线互连的最 佳方案, 但在高频信号传输的环境中, 电生长铜层的表 面粗鋉度直接影响信号传输的损耗. 因此, 本文研究了 含不同有机添加剂的镀液体系对铜电生长粗䊅度的影 响, 采用模拟仿真和电化学测试分析有机添加剂在电 生长过程中的作用机理, 进而解释电生长粗糙度差异 的原因; 同时，考察了铜电生长粗粘度差异对信号传 输损耗的影响.

\section{2 实验部分}

\section{1 有机添加添加剂的分子动力学和量子化学计算}

选取 $\mathrm{Cu}(111)$ 晶面作为反应界面，将优化过后的 SPS、聚氧乙烯-聚丙乙烯嵌段共聚物(Ethylene oxidepropylene oxide copolymer, EO/PO)和健那绿(Janus Green B, JGB)的分子随机放置于盒子中, 模拟真实的 铜电生长环境. 最后将含有添加剂和水分子的不定型 盒子与先前构造的超晶胞结构配合, 就完成了搭建动 力学模拟的实验环境. 通过Material Studio(MS)软件分 别对电沉积铜添加剂SPS、EO/PO、JGB分子进行吸 附行为模拟.

对铜电生长有机添加剂SPS、EO/PO和JGB进行 分子动力学模拟, Forcite动力学计算模块的数值求解 方法为有限差分方法，由于电生长过程中系统能量可 能有起伏但系综温度保持恒定，离子系综选择正则系 综, 力场选择为COMPASS力场. 根据小分子选用小机 组计算的原则, 量子化学计算过程的原子基组选择6-
311G，使用B3LYP模块方法进行密度泛函理论计算， 溶液体系选择默认的水体系, 计算类型为分子优化计 算，收玫准则选择高精度，对有机添加剂分别进行 计算.

计算有机添加剂的最高占有分子轨道(Highest occupied molecular orbital, HOMO)和最低未占据分子轨 道(Lowest unoccupied molecular orbital, LUMO)和 LUMO-HOMO能隙 $(\Delta E)$, 用以解释有机物与金属表面 在原子水平上的相互作用机制。分子的 HOMO 、 LUMO大小分别由电离势IE、电子亲合能EA来确定, LUMO-HOMO能隙由HOMO、LUMO确定, 如公 式(1 3):

$\Delta E=E_{\mathrm{LOMO}}-E_{\text {Номо }}$,

$\mathrm{EA}=-E_{\text {номо }}$,

$\mathrm{IE}=-E_{\mathrm{LOMO}}$,

根据电离式和电子亲和能的数据可以得出分子的 绝对电负性 $\chi$, 其计算如公式(4):

$\chi=(\mathrm{IE}+\mathrm{EA}) / 2$.

\section{2 有机添加剂的电化学分析}

为了研究三种添加剂对铜结晶形貌的作用机理, 分别做了不同添加剂体系下的恒电流测试和循环伏安 测试. 该实验在Autolab电化学工作站(PGSTAT 302N, 瑞士万通)进行, 电极体系包括对电极、参比电极、工 作电极. 其中对电极为铜棒, 置于注满电镀液的玻璃管 中, 并用多孔陶瓷材料将玻璃管封底; 参比电极采用饱 和录一硫酸亚录电极(SSE), 置于鲁金毛细管中, 工作电 极采用直径为 $5 \mathrm{~mm}$ 的铂-旋转圆盘电极 (Rotating disk electrode, RDE); 电化学测试装置在 $25^{\circ} \mathrm{C}$ 水浴环境下 进行，镀液体积为 $200 \mathrm{~mL}$, 实验中的电位都是相对于 SSE参比电极的相对电位. 基础镀液 (Virgin-made solution，VMS)的成分包括 $75 \mathrm{~g} / \mathrm{L}$ 五水硫酸铜、 $220 \mathrm{~g} / \mathrm{L}$ 硫 酸、 $60 \mathrm{mg} / \mathrm{L}$ 氯离子, 而VMS中加入有机添剂加剂及 含量为: $4 \mathrm{mg} / \mathrm{L}$ SPS、 $400 \mathrm{mg} / \mathrm{L} \mathrm{EO} / \mathrm{PO}$ 和 $4 \mathrm{mg} / \mathrm{L} \mathrm{JGB}$; 阳极使用钛网阳极, 并用滤纸包覆, 施加 $2 \mathrm{~A} / \mathrm{dm}^{2}$ 的直 流电流, 整个电沉积过程由空气搅拌器鼓气搅拌, 气流 量 $1 \mathrm{~L} / \mathrm{min}$, 哈林槽置于恒温水浴中, 维持镀液温度在 $25^{\circ} \mathrm{C}$ 条件下电镀 $30 \mathrm{~min}$. 不同镀液体系的恒电流测试 条件: 将Pt-RDE以 $200 \mathrm{~A} / \mathrm{m}^{2}$ 的电流密度在VMS中电沉 积 $500 \mathrm{~s}$, 将得到的 $\mathrm{Cu}-\mathrm{RDE}$ 作为恒电流测试的工作电 
极, 设定电流密度为 $200 \mathrm{~A} / \mathrm{m}^{2}$, 旋转圆盘电极转速为 $1500 \mathrm{r} / \mathrm{min}$. 不同镀液体系的循环伏安测试条件: 扫描 方向为从开路电位往负方向扫到 $-0.7 \mathrm{~V} v \mathrm{~s}$. SSE, 然后 正向扫描到 $0.4 \mathrm{~V} v s$. SSE, 再反向扫描回开路电位.

考察电镀体系及无电场体系下不同镀液的接触角 变化, 为了测量不同镀液体系下的接触角大小及电沉 积过程接触角的变化, 本实验使用了视频光学接触角 测定仪(JY-PHa, 承德优特检测仪器制造有限公司). 镀 液的添加剂体系施加电流(电流密度为 $200 \mathrm{~A} / \mathrm{m}^{2}$ ), 接触 角测量时间为 $60 \mathrm{~s}$.

\section{3 电生长铜面的表征测试}

电生长铜面的微观形貌及粗粘度用扫描电子显微 镜(Scanning electron microscope, SEM) (S3400, 日立公 司)和3D激光共焦显微镜(OSL4100，奥林巴斯公司)观 测, 晶格生长情况用 X射线衍射仪(X-ray diffraction, XRD) (X'PERT MPD, 飞利浦公司)测试. 设计出测试 板, 采用插入损耗方法表征电生长铜面粗糙度对信号 传输损耗的影响.

\section{3 结果与讨论}

\section{1 含不同有机添加剂镀液的铜电生长效果}

沉积含不同添加剂镀液的铜电生长表面微观形貌 用SEM和3D激光共焦显微镜观测, 结果如图1所示. 在
无添加剂的情况下, 如图 1a-1和a-2, 铜结晶形貌的结晶 形状不规则, 表面凹凸不平, 铜面不存在孔隙, 说明在 没有添加剂的体系中, 不能获得平整化的铜生长效果. 在只含有SPS的镀液中, 如图 1b-1和b-2, 铜面结晶近似 棱雉结构, 结晶颗粒较大, 铜结晶颗粒分布在 $2 \sim 8 \mu \mathrm{m}$, 结晶形貌有空洞、部分存在孔隙, 其铜生长的结晶颗 粒更大, 结晶不细密, 粗粘度更大. 仅含有 $\mathrm{EO} / \mathrm{PO}$ 的情 况下, 如图1c-1和c-2, 许多亚微米级别的结晶颗粒遍布 在整个铜面, 其结晶颗粒比无添加剂时较小, 但仍有 $5 \mu \mathrm{m}$ 以上的结晶存在. 仅含有JGB的情况下, 如图 $1 \mathrm{~d}-1$ 和d-2, 其表面结晶形貌分布杂乱, 结晶颗粒在 $1 \sim 6 \mu \mathrm{m}$, 相比没有添加剂存在时, 该铜面出现了长条状结晶. 但 是，当镀液同时加入SPS、JGB、EO/PO时，电生长铜 面表现为非常平整, 如图1e-1和e-2.

根据IPC-4562标准, 用 $R_{\mathrm{z}}$ 值描述铜䈃的粗粘度. 有 机添加剂在铜电生长过程发挥着不同的作用, 进而影 响铜颗粒生长平整度, 含不同添加剂镀液的铜电生长 表面粗糙度如图2 $\mathrm{a}$ 所示. 当镀液中无添加剂时, 电生 长铜面的 $R_{\mathrm{z}}$ 值为 $2.85 \mu \mathrm{m}$; 与无添加的镀液相比, 加入 $\mathrm{SPS}$ 的镀液, 电生长铜面的 $R_{\mathrm{z}}$ 值迅速增大至 $4.88 \mu \mathrm{m}$; 只 含 $\mathrm{EO} / \mathrm{PO}$ 的镀液, 电生长铜面的 $R_{\mathrm{z}}$ 值有所降低, 为 $1.94 \mu \mathrm{m}$; 而只含 $\mathrm{JGB}$ 的镀液, 则电生长铜面的 $R_{\mathrm{z}}$ 值有 所升高, 为 $3.23 \mu \mathrm{m}$. 但是, 当SPS、 EO/PO和JGB同时 加入VMS时, 电生长铜面的 $R_{\mathrm{z}}$ 值小至 $0.22 \mu \mathrm{m}$, 与图 $1 \mathrm{e}-$ 1 和e-2的结果一致. 从图 $2 \mathrm{~b}$ 也可以看出, 含不同添加剂
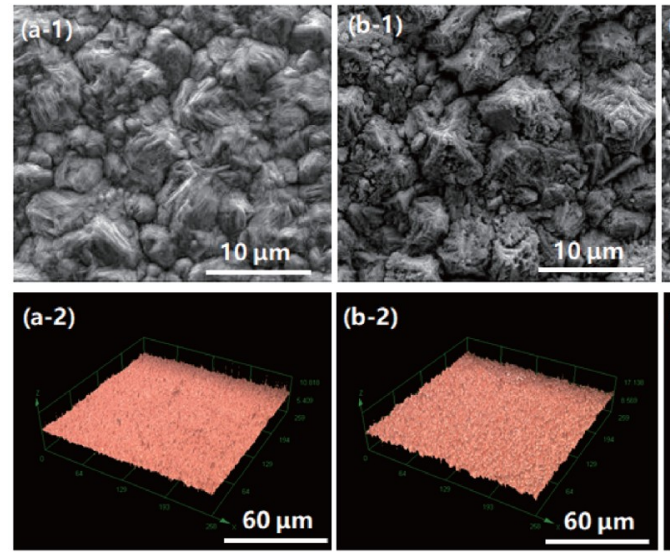
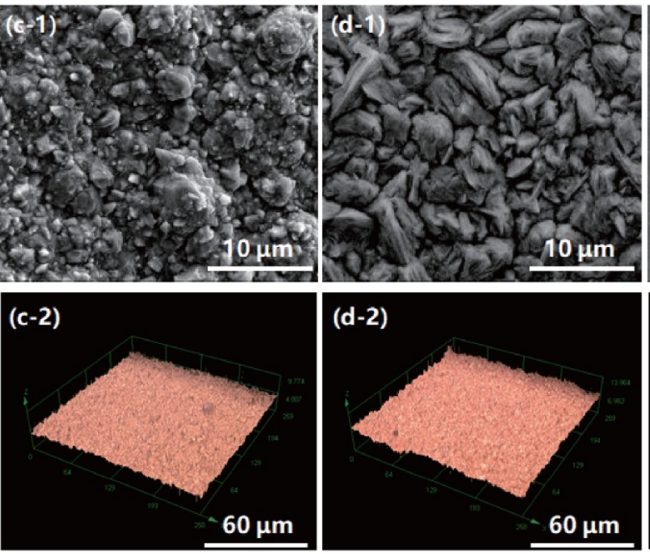
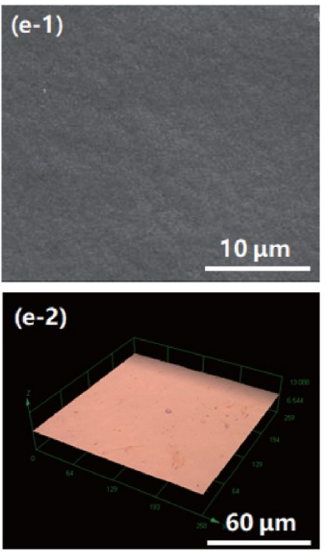

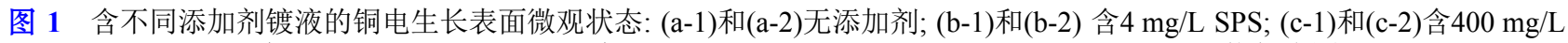
$\mathrm{EO} / \mathrm{PO}$; (d-1)和(d-2)含 $4 \mathrm{mg} / \mathrm{L} \mathrm{JGB}$; (e-1)和(e-2)含 $4 \mathrm{mg} / \mathrm{L} \mathrm{SPS} 、 400 \mathrm{mg} / \mathrm{L} \mathrm{EO} / \mathrm{PO}$ 和 $4 \mathrm{mg} / \mathrm{L} \mathrm{JGB}$ (网络版彩图)

Figure 1 The surface microstructure of copper growth in plating solutions containing different additives: (a-1) and (a-2) no additive; (b-1) and (b-2) containing $4 \mathrm{mg} / \mathrm{L}$ SPS; (c- 1) and (c-2) containing $400 \mathrm{mg} / \mathrm{L}$ EO/PO; (d-1) and (d-2) containing $4 \mathrm{mg} / \mathrm{L} \mathrm{JGB}$; (e-1) and (e-2) containing $4 \mathrm{mg} / \mathrm{L}$ SPS, $400 \mathrm{mg} / \mathrm{L} \mathrm{EO} / \mathrm{PO}$ and $4 \mathrm{mg} / \mathrm{L}$ JGB (color online) 

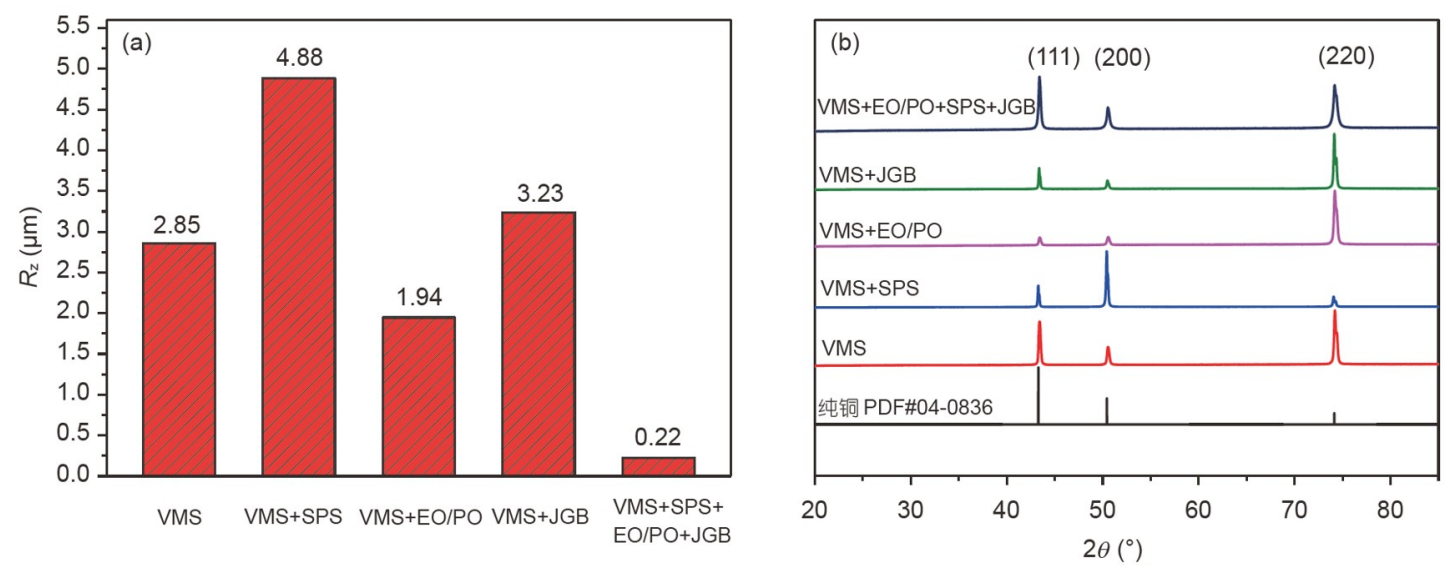

图 2 (a) 含不同添加剂镀液的铜电生长表面粗粘度和(b) XRD曲线 (网络版彩图)

Figure 2 Surface roughness (a) and XRD patterns (b) of copper electro-grown in plating solutions containing different additives (color online).

镀液的铜电生长晶格取向也有所不同. 含SPS的镀液, 铜晶格的优先生长方向发生在(200), 而含 $\mathrm{EO} / \mathrm{PO}$ 或 $\mathrm{JGB}$ 的镀液，则优先发生在(200)方向. 当在VMS中同 时加入SPS、 EO/PO和JGB，最优生长方向为(111)和 (200). 其原因可能是有机添加剂间存在复杂的相互作 用，每种添加剂依靠自身均无法起到有效的整平作用， 但组合加入后则发挥了有效的整平作用，即有机添加 剂之间存在复杂的协同或拮抗作用.

\section{2 有机添加剂的电子特性及分子轨道研究}

量子化学计算得到的HOMO、LUMO如图3所示, 同时可得到各自的HOMO、LUMO能量值，见表1. 根 据前线轨道理论, $\mathrm{HOMO}$ 上的电子能量最高, 最容易发 生变动而失去, LUMO在未占有轨道中能量最低, 更容 易接受电子, 所以 $E_{\mathrm{HOMO}}$ 越大的添加剂更容易贡献电 子, $E_{\mathrm{LOMO}}$ 越小的添加剂越容易接受电子. $\Delta E$ 的大小反 映的是电子从最高占据轨道跃迁到空轨道的能力，在 一定程度上可以反映添加剂分子与其他分子或离子发 生反应的可能性.

如表1所示, $\mathrm{EO} / \mathrm{PO}$ 的 $E_{\mathrm{HOMO}}$ 值为 $-8.18 \mathrm{eV}, \mathrm{SPS}$ 的 $E_{\mathrm{HOMO}}$ 值为 $-6.67 \mathrm{eV}, \mathrm{JGB}$ 的 $E_{\mathrm{HOMO}}$ 值为 $-5.15 \mathrm{eV}$, 在三 种有机添加剂中 $\mathrm{EO} / \mathrm{PO}$ 的 $E_{\mathrm{HOMO}}$ 值最小, 所以与 $\mathrm{SPS}$ 和 $\mathrm{JGB}$ 分子相比, $\mathrm{EO} / \mathrm{PO}$ 最不容易发生电子的贡献, 并且 $\mathrm{EO} / \mathrm{PO}$ 的 $E_{\mathrm{LOMO}}$ 为 $0.69 \mathrm{eV}$, 均大于 $\mathrm{SPS} 、 \mathrm{JGB}$ 分子, 即 最不容易发生电子的失去，且三种有机添加剂的 $\Delta E$ 从大到小为 $\mathrm{EO} / \mathrm{PO} 、 \mathrm{SPS} 、 \mathrm{JGB}$, 所以推测 $\mathrm{EO} / \mathrm{PO}$ 在
电沉积过程中性质比较稳定, 不容易发生电子转移及 化学成键. 而对于 JGB分子, 它的 $E_{\text {HOMO }}$ 值最大, $E_{\mathrm{LOMO}}$ 值最小, 这说明在电沉积过程中JGB发生电子转移的 可能性更大, 更容易发生化学反应, 这可能是JGB在电 沉积过程中发生分解反应进而形成反馈 $\pi$ 键的缘故 ${ }^{[10]}$. 另外, 从表 1 可知, 有机添加剂的电负性值结果为 $\mathrm{JGB}>\mathrm{SPS}>\mathrm{EO} / \mathrm{PO}$, 而电负性 $\chi$ 被认为是化学反应理论 中分子的重要参数, 是对分子整体电性质的度量. 根 据桑德森的电负性平衡理论 ${ }^{[11]}$ : 两种物质的电负性差 异越小, 越容易产生更快的吸附平衡. 因此, 可推测三 种有机添加剂的反应活性从JGB、SPS到 $\mathrm{EO} / \mathrm{PO}$ 依次 降低, 即 $\mathrm{EO} / \mathrm{PO}$ 能更能持续稳定地存在于铜面.

图4是SPS、EO/PO、JGB三种分子在经历 $200 \mathrm{ps}$ 动力学模拟后的吸附结果. 在电生长反应过程中, 三种 有机分子均移动到了铜面附近，表明它们均能够在表 面被吸附. 其中 SPS 分子的- $\mathrm{SO}_{3}$-基团被优先吸附在铜 面, 说明SPS分子可能是优先以- $\mathrm{SO}_{3}$-基团与铜面成键, 这与Dow的研究结果保持一致 ${ }^{[12]}$; JGB分子几乎平行 贴于铜面，由于其环状结构的存在，该区域的 $\mathrm{Cu}$ 表面 完全被覆盖, 这可能会使在该区域 $\mathrm{Cu}^{2+}$ 的还原受到阻 碍, 进而对局部区域起到抑制作用 ${ }^{[13]}$; 图3的量子化学 计算结果已知 $\mathrm{EO} / \mathrm{PO}$ 可持续稳定在铜层, 且其作为较 大的分子，吸附在阴极表面后，会阻挡铜离子还原的 活性位点, 阻碍铜离子的传质过程, 进而对铜沉积起 到抑制作用，减缓铜的生长速度，对形成较低粗粘度 的铜面具有积极作用. 

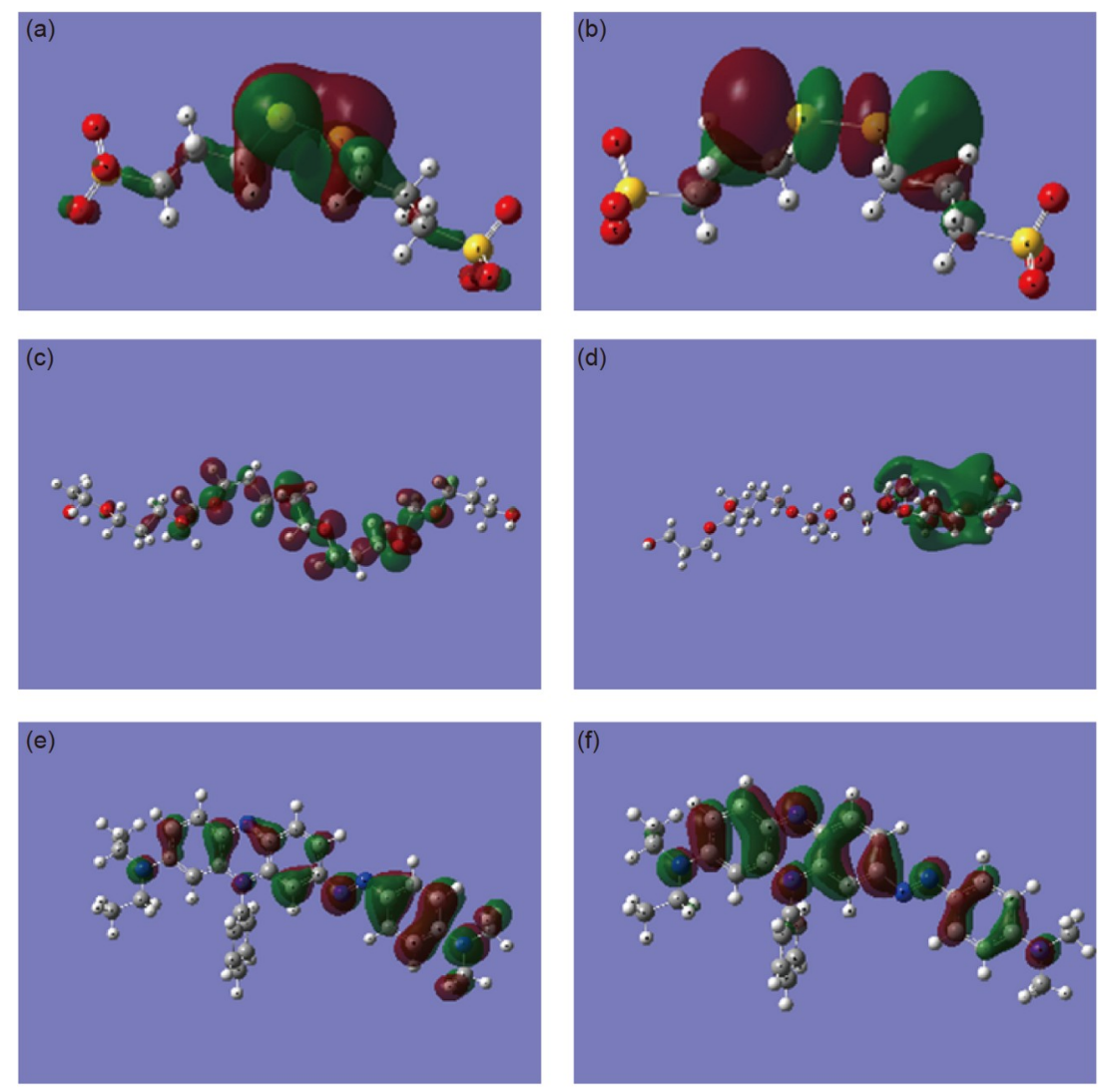

图 3 不同有机添加剂的量子化学计算HOMO和LUMO结果: (a) SPS的HOMO; (b) SPS的LUMO; (c) EO/PO的HOMO; (d) EO/PO 的LUMO; (e) JGB的HOMO; (f) JGB的LUMO (网络版彩图)

Figure 3 Quantum chemical calculation results of HOMO and LUMO of different organic additives: (a) HOMO of SPS; (b) LUMO of SPS; (c) HOMO of EO/PO; (d) LUMO of EO/PO; (e) JGB HOMO; (f) LUMO of JGB (color online).

表 1 SPS、EO/PO、JGB分子的HOMO、LUMO轨道能量 Table 1 HOMO and LUMO energies of SPS, EO/PO, and JGB molecules

\begin{tabular}{cccc}
\hline 有机添加剂类别 & SPS & EO/PO & JGB \\
\hline HOMO $(\mathrm{eV})$ & -6.67 & -8.18 & -5.15 \\
LUMO $(\mathrm{eV})$ & -1.02 & 0.69 & -3.63 \\
$\mathrm{IE}(\mathrm{eV})$ & 6.67 & 8.18 & 5.15 \\
$\mathrm{EA}(\mathrm{eV})$ & 1.02 & -0.69 & 3.63 \\
$\Delta E(\mathrm{eV})$ & 5.64 & 8.87 & 1.51 \\
$\chi$ & 7.70 & 7.48 & 8.78 \\
\hline
\end{tabular}

\section{3 有机添加剂镀液体系的电化学分析}

无任何添加剂的VMS 在铜面上的润湿性最差，达 到 $59.4^{\circ}$, 这是因为铜面不含有羟基, 不含添加剂的电 镀液在纯铜表面的润湿性较差 ${ }^{[14]}$. 电场的引入会引起
镀液体系中添加剂的吸附, 进而影响镀液的润湿性. 在无电流和有电电流的条件下，含不同有机添加剂的 镀液体系静置 $60 \mathrm{~s}$ 后的接触角如图5. 在施加电场后, SPS体系的接触角从 $55.0^{\circ}$ 增加 $59.0^{\circ}, \mathrm{EO} / \mathrm{PO}$ 体系的接 触角从 $48.8^{\circ}$ 增加到 $56.7^{\circ}$, 这可能是存在电流时添加剂 的吸附作用减弱 ${ }^{[15]}$, 相应的润湿性有一定减小. JGB体 系的接触角从 $56.0^{\circ}$ 减小到 $54.8^{\circ}$, 变化较小, 可能因为 JGB单独存在时对润湿性的影响不明显. 其中接触角 最小的添加剂体系为SPS-EO/PO-JGB体系, 接触角从 $49.4^{\circ}$ 减小到 $41.8^{\circ}$, 说明该体系在电场作用下有更低的 表面张力、更好的润湿性, 在沟壑、不平整的部位能 够充分润湿, 可能具有更好的整平性能.

由图6a恒电流曲线可知, 在 VMS中加入 $\mathrm{EO} / \mathrm{PO}$ 后, 溶液电势从 $-0.53 \mathrm{~V}$ 瞬间下降到 $-0.73 \mathrm{~V}$, 说明 $\mathrm{EO} / \mathrm{PO}$ 能够强烈地抑制铜的沉积, 然后 $\mathrm{EO} / \mathrm{PO}$ 的吸附/解离逐 渐达到平衡, 稳定在 $-0.73 \mathrm{~V}$ 附近. 根据图 4 对 $\mathrm{EO} / \mathrm{PO}$ 的 

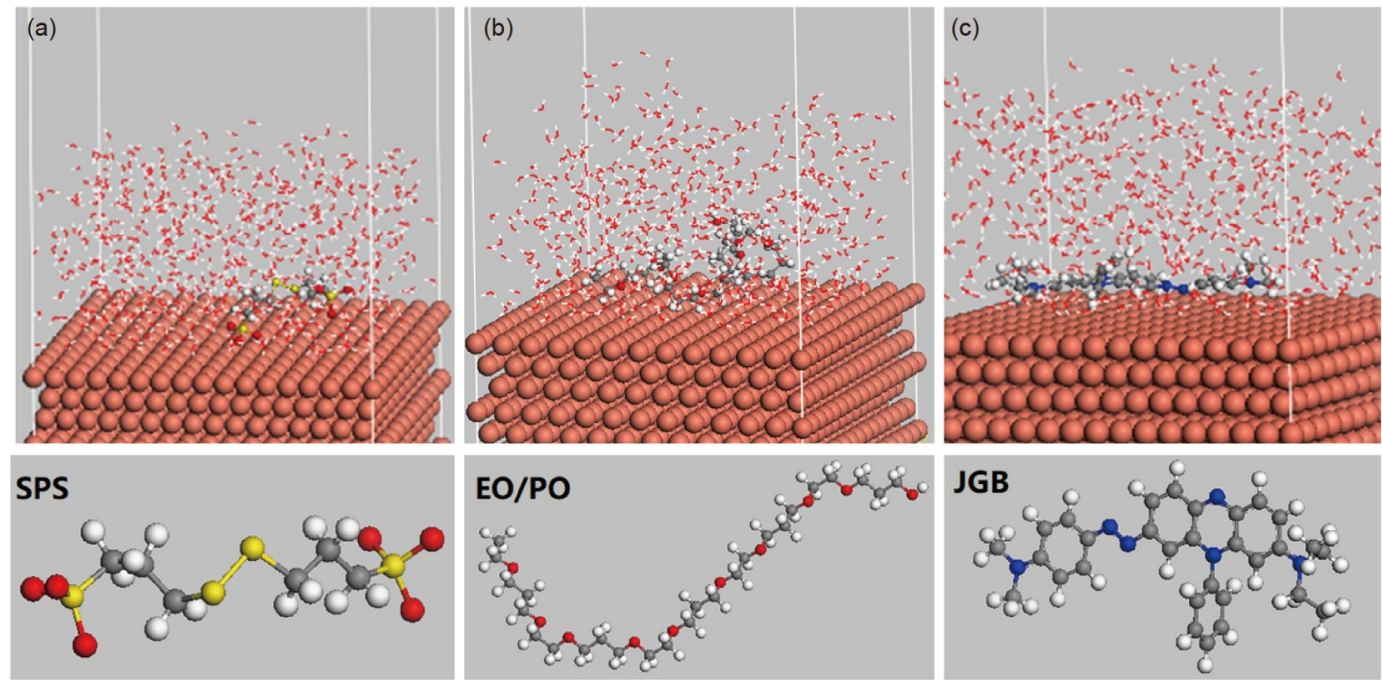

图 4 不同有机添加剂的吸附仿真结果: (a) SPS; (b) EO/PO; (c) JGB (网络版彩图)

Figure 4 The adsorption simulation results of different organic additives: (a) SPS; (b) EO/PO; (c) JGB (color online).
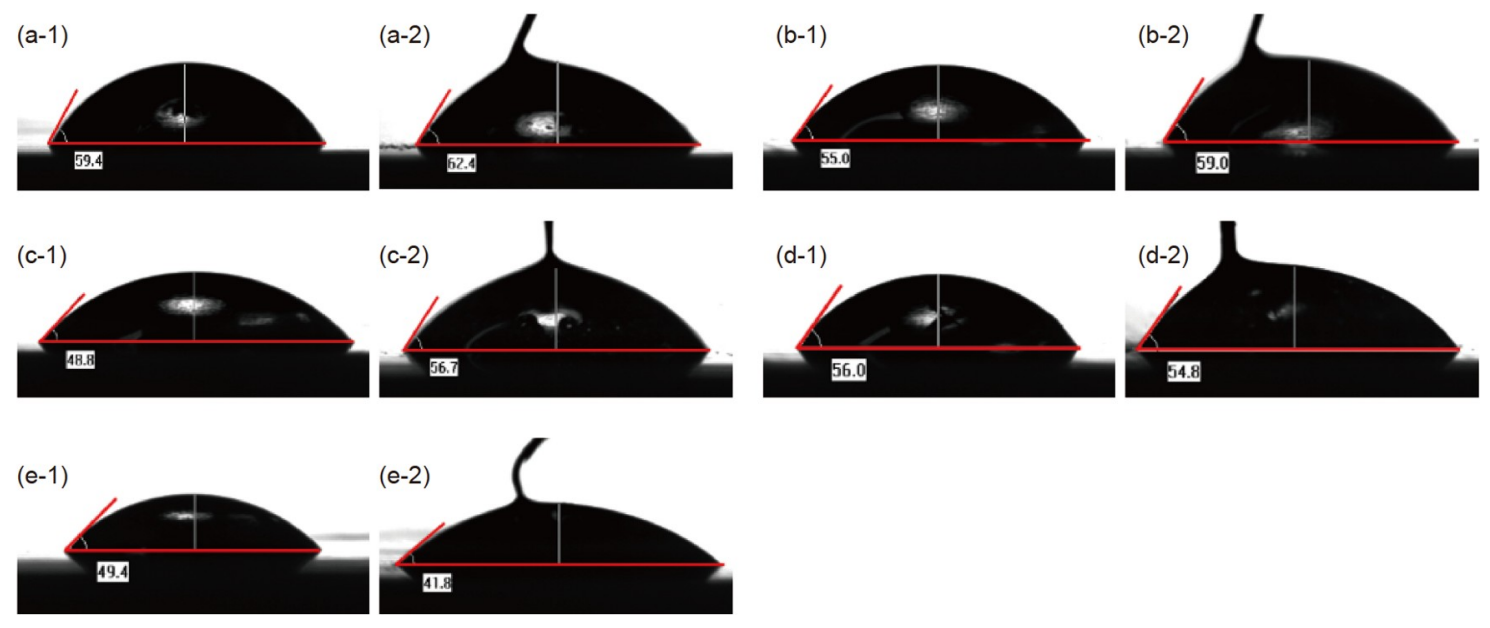

图 5 含不同添加剂镀液体系的接触角: (a-1) 无添加剂, 无电流; (a-2) 无添加剂, 通 $200 \mathrm{~A} / \mathrm{m}^{2}$ 电流; (b-1) SPS, 无电流; (b-2) SPS, 通 $200 \mathrm{~A} / \mathrm{m}^{2}$ 电流; (c-1) EO/PO, 无电流; (c-2) EO/PO, 通200A/ $\mathrm{m}^{2}$ 电流; (d-1) JGB, 无电流; (d-2) JGB, 通 $200 \mathrm{~A} / \mathrm{m}^{2}$ 电流; (e-1) SPS $+\mathrm{EO} / \mathrm{PO}+\mathrm{JGB}$, 无电流; (e-2) SPS+EO/PO+JGB, 通 $200 \mathrm{~A} / \mathrm{m}^{2}$ 电流 (网络版彩图)

Figure 5 Contact angles of plating solution systems containing different additives: (a-1) no additives, with no current; (a-2) no additives, at $200 \mathrm{~A} / \mathrm{m}^{2}$; (b-1) SPS, with no current; (b-2) SPS, at $200 \mathrm{~A} / \mathrm{m}^{2} ;(\mathrm{c}-1) \mathrm{EO} / \mathrm{PO}$, with no current; (c-2) EO/PO, at $200 \mathrm{~A} / \mathrm{m}^{2} ;$ (e) JGB, with no current; (e) $\mathrm{JGB}$, at $200 \mathrm{~A} / \mathrm{m}^{2}$; (e) SPS+EO/PO+JGB, with no current; (e) SPS+EO/PO+JGB, at $200 \mathrm{~A} / \mathrm{m}^{2}$ (color online).

仿真吸附行为研究，该结果与 $\mathrm{EO} / \mathrm{PO}$ 具有较大的结合 能有关，EO/PO吸附在阴极阻挡了反应活性位点进而 抑制了铜沉积. 在含有 $\mathrm{Cl}^{-}$的VMS中, 溶液中阴极附近 的 $\mathrm{Cl}^{-}$能够与 $\mathrm{EO} / \mathrm{PO}$ 结合并在反应界面形成抑制层, 在 电沉积环境中阻碍 $\mathrm{Cu}^{2+}$ 和 $\mathrm{Cu}^{+}$向电极表面迁移 ${ }^{[15,16]}$, 而 该抑制层的形成增加了阴极表面的粘度，产生了EO/
PO的强烈极化作用, 图6b循环伏安曲线III的最小铜剥 离峰也进一步证明 $\mathrm{EO} / \mathrm{PO}$ 的抑制铜生长行为. 铜电生 长速率在强抑制作用下较低, 铜在各个晶面的生长速 度达到一致 ${ }^{[17]}$ ，且抑制剂的存在有利于更小的晶核产 生 ${ }^{[18]}$. 在500 s 处, 在含有 $\mathrm{EO} / \mathrm{PO}$ 的VMS中加入 SPS后, 镀液的电势从 $-0.73 \mathrm{~V}$ 开始逐渐上升到 $-0.67 \mathrm{~V}$, 表现 
出去极化效应 ${ }^{[19]}$, 图6b循环伏安曲线II的最大铜剥离 峰也说明了SPS的铜电生长加速行为. 这是因为 $\mathrm{EO} /$ PO虽然在体系中形成了抑制层，但抑制层被SPS持续 破坏, 同时在被破坏的位置被SPS吸附并取代 ${ }^{[20]}$. 镀液 继续加入 JGB, 镀液电势再次下降, 即JGB同样具有极 化作用, 抑制铜的生长, 最终电势稳定在 $-0.71 \mathrm{~V}$ 附近. 在同时含SPS、EO/PO和JGB的VMS中, 由于添加剂之 间的竞争吸附行为, 图6b循环伏安曲线 $\mathrm{V}$ 的铜剥离峰 介于较大与最小之意，即有机添加间的协同作用让铜 晶粒形成和长大更加细化，这可能就是造成图 $1 \mathrm{e}-1$ 表

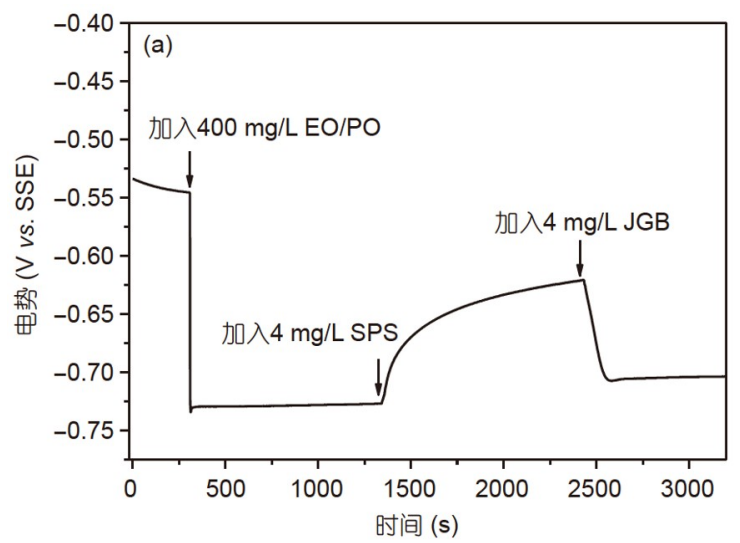

现出铜面生长最平整的原因.

\section{4 信号传输损耗测试与分析}

信号传输损耗测试用的电路结构及测试板如图 7a 和 $\mathrm{b}$ ，测试板长 $140 \mathrm{~mm}$ 、宽 $24 \mathrm{~mm}$ 、板厚 $10 \mathrm{~mm}$ ，信号 层由 $254 \mathrm{~mm}$ 长线和 $127 \mathrm{~mm}$ 短线的差分线组构成, 使 用插入损耗方法测试信号传输损耗. 用含不同有机添 加剂的镀液在测试板的传输线路进行表面铜电生长, 条件如下: 电流密度为 $200 \mathrm{~A} / \mathrm{m}^{2}$, 空气摚拌器鼓气流量 为 $1 \mathrm{~L} / \mathrm{min}$, 阳极使用滤纸包覆的钛网阳极, 在 $25^{\circ} \mathrm{C}$ 的

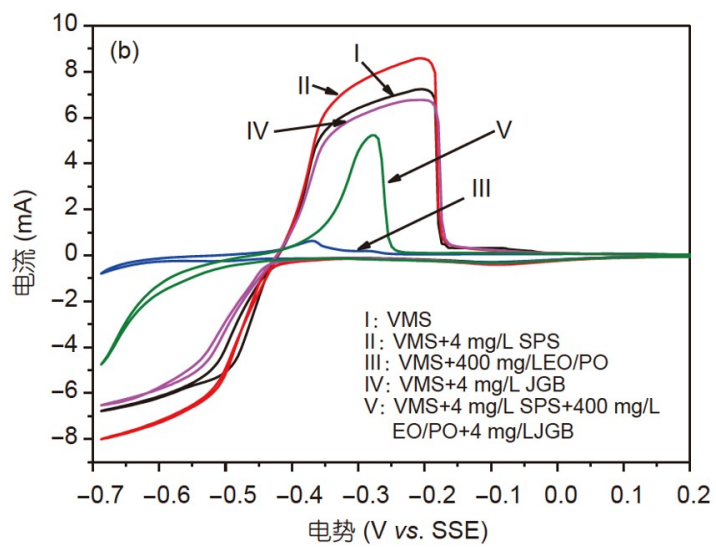

图 6 VMS加入不同有机添加剂的恒电流曲线(a)和循环伏安曲线(b) (网络版彩图)

Figure 6 Constant current curve (a) and cyclic voltammetry curves (b) of VMS with different organic additives (color online).
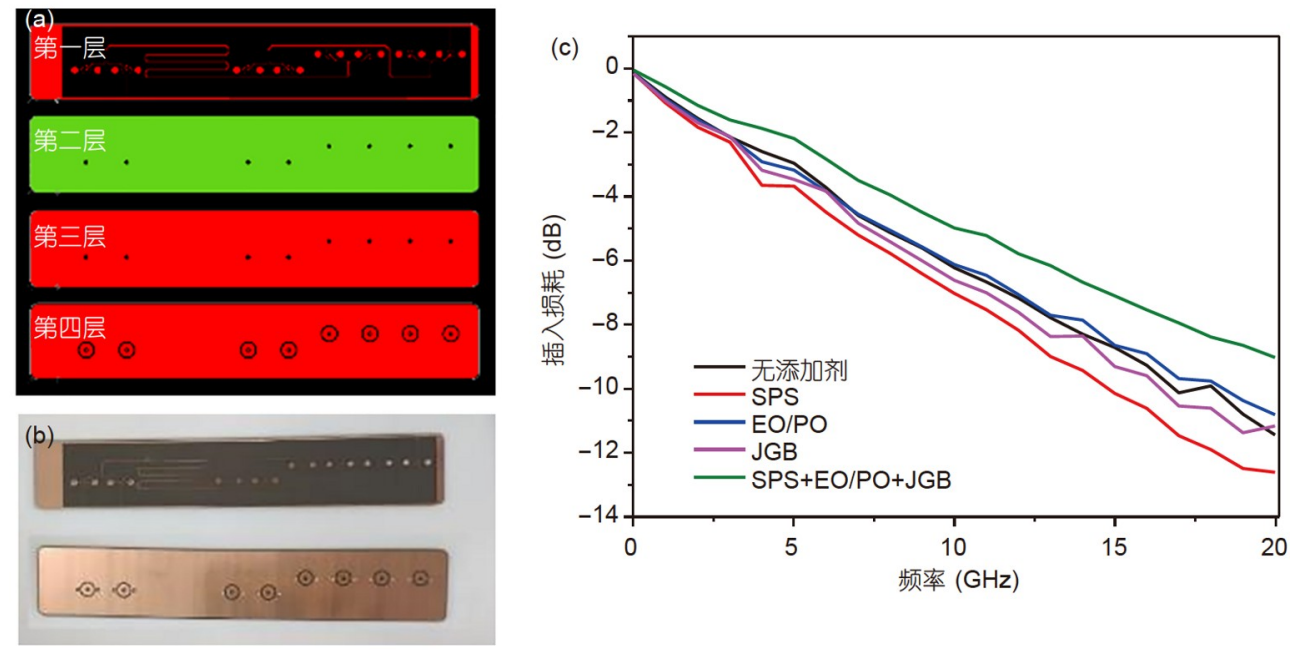

图 7 测试板电路结构(a)、制作的测试板(b)和测试板传输线路在不同添加剂镀液电生长铜后的插入损耗结果(c) (网络版彩 图)

Figure 7 Test board circuit structure (a), produced test board (b) and test board transmission line insertion loss results after electro-growing copper in different additive plating solutions (c) (color online). 
恒温水浴中电镀 $30 \mathrm{~min}$. 根据图1可知，不同添加剂镀 液会产生不同表面形貌的铜层，测试板电生长铜后进 行插入损耗测试，结果如图7c. 无添加剂镀液电生长 铜层后，线路的插入损耗值为 $-11.4 \mathrm{~dB} @ 20 \mathrm{GHz}$; 含 SPS的镀液电生长铜层后, 线路的插入损耗值最大, 达 到-12.6 dB@20 GHz, 而含SPS、EO/PO和JGB体系的 插入损耗值最小, 为 $-8.9 \mathrm{~dB} @ 20 \mathrm{GHz}$. 图7c的综合比 较可知, 根据VMS所加入的有机添加剂种类排序，插 入损耗值从大到小是 $\mathrm{SPS}>\mathrm{JGB}>\mathrm{EO} / \mathrm{PO}>\mathrm{SPS}-\mathrm{EO} / \mathrm{PO}-$ $\mathrm{JGB}$ ，而图2所测的电生长铜表面粗䊁度从大到小是 $\mathrm{SPS}>\mathrm{JGB}>\mathrm{EO} / \mathrm{PO}>\mathrm{SPS}-\mathrm{EO} / \mathrm{PO}-\mathrm{JGB}$, 粗粘度与损耗值 的顺序能够很好地对应，即插入损耗值的增大是由传 输线粗粘度变大而引起的. 因此，使用具有优异整平 效果的有机添加剂体系，可有效降低信号传输的插入 损耗值, 保障互连结构的信号完整性.

\section{4 结论}

通过研究有机添加剂SPS、JGB、EO/PO及三者 组合对铜面电生长粗鋉度的影响，结果表明含三者组 合的VMS 具有最优的铜面粗粘度调控效果，即电生长 铜面的 $R_{z}$ 值小至 $0.22 \mu \mathrm{m}$. 采有量子化学与分子动力学 仿真、通电接触角测试和电化学分析测试, 研究了有 机添加剂的吸附行为及三者的各自差异和协同作用， 进而得出低粗粘度铜面电生长的原因是有机添加剂 SPS、JGB、EO/PO之间相互竞争吸附的调控，从而 抑制铜颗粒生长的速率，并由含三者的VMS 电生长 出表面形貌平整的铜层，实现了最小插入损耗，即 $-8.9 \mathrm{~dB} @ 20 \mathrm{GHz}$. 因此, 采用多元复合有机添加剂体 系, 可调控电生长出表面形貌平整的铜层, 保障互连结 构在高频信号传输的完整性.

\section{参考文献}

1 He W, Wang S, Chen Y. Advanced Technology of Printed Circuit and Printed Electronics. Beijing: Science China Press, 2016 (in Chinese) [何为, 王守绪, 陈苑明. 印制电路与印制电子先进技术(下册). 北京: 科学出版社, 2016]

2 Wang C, Wen N, Zhou G, Wang S, He W, Su X, Hu Y. Appl Surf Sci, 2017, 422: 738-744

3 Chen Y, Gao Y, Jin X, Zhou X, Wang S, He W, Hong Y, Zhou G, Zhang W, Sun R, Huang Y, Tang Y. J Mater Sci-Mater Electron, 2019, 30: $16226-16233$

4 Chan PF, Ren RH, Wen SI, Chang HC, Dow WP. J Electrochem Soc, 2017, 164: D660-D665

5 Sudibyo, How MB, Aziz N. IOP Conf Ser-Mater Sci Eng, 2018, 285: 012021

6 Rodchanarowan A, Free ML. Mater Trans, 2012, 53: 1695-1698

7 Woo TG, Park IS, Lee HW, Seol KW. Korean J Mater Res, 2006, 16: 710-714

8 Zhang W, Lu X, Liu Y, Luo J. J Electrochem Soc, 2007, 154: D526

9 Gu XQ, Xu SS. China Integrated Circuit, 2008, 08: 61-64 (in Chinese) [顾晓清, 徐赛生. 中国集成电路, 2008, 08: 61-64]

10 Lai Z, Wang S, Wang C, Hong Y, Zhou G, Chen Y, He W, Peng Y, Xiao D. Comput Mater Sci, 2018, 147: 95-102

11 Geerlings P, De Proft F. IJMS, 2002, 3: 276-309

12 Dow WP, Chiu YD, Yen MY. $J$ Electrochem Soc, 2009, 156: D155

13 Wang C, Zhang J, Yang P, An M. Electrochim Acta, 2013, 92: 356-364

14 Liu CW, Tsao JC, Tsai MS, Wang YL. J Vacuum Sci Tech A-Vacuum Surfs Films, 2004, 22: 2315-2320

15 Feng ZV, Li X, Gewirth AA. J Phys Chem B, 2003, 107: 9415-9423

16 Xiao N, Li D, Cui G, Li N, Li Q, Wu G. Electrochim Acta, 2014, 116: 284-291

17 Bozzini B, Fanigliulo A, Serra M. J Cryst Growth, 2001, 231: 589-598

18 Manu R, Jayakrishnan S. J Electrochem Soc, 2009, 156: D215

19 Huynh TMT, Weiss F, Hai NTM, Reckien W, Bredow T, Fluegel A, Arnold M, Mayer D, Keller H, Broekmann P. Electrochim Acta, 2013, 89: $537-548$

20 Huang Q, Baker-O’Neal BC, Parks C, Hopstaken M, Fluegel A, Emnet C, Arnold M, Mayer D. J Electrochem Soc, 2012, 159: D526-D531 


\title{
Investigation of organic additives to regulate growth and signal transmission loss of copper interconnection structure
}

\author{
Yuanming Chen ${ }^{1,2}$, Jiaying Xu ${ }^{1}$, Lingzhi Zhang ${ }^{1}$, Xiaohuang Zhuo ${ }^{1}$, Yunzhao Chen ${ }^{1}$, Chong Wang ${ }^{1}$, \\ Wei $\mathrm{He}^{1,2^{*}}$, Shouxu Wang ${ }^{1}$, Weihua Zhang ${ }^{2}$, Xinhong $\mathrm{Su}^{2}$, Yuyao Luo ${ }^{2}$ \\ ${ }^{1}$ School of Materials and Energy, University of Electronic Science and Technology of China, Chengdu 610054, China \\ ${ }^{2}$ Zhuhai Founder Sci-Tech High-density Electronics Co., Ltd and Zhuhai Founder Sci-Tech Multilayer Circuit Board Co., Ltd, Zhuhai 519175, China \\ *Corresponding author (email: heweiz@uestc.edu.cn)
}

\begin{abstract}
Signal integrity is severely limited by the copper roughness. Plating solution containing different organic additives could influence the roughness of electroplating copper growth. Molecular simulation and electrochemical tests were used to regulate the competitive adsorption behavior of organic additives on the copper surface and understand the possible mechanism on the difference of copper growth. The results show that the plating solution system containing bis-(sodium sulfopropyl)-disulfide (SPS), ethylene oxide-propylene oxide copolymer (EO/PO) and Janus Green B (JGB) can contribute to the copper growth with smooth surface, leading to insertion loss of $-8.9 \mathrm{~dB} @ 20 \mathrm{GHz}$. Therefore, organic additives could be used to regulate the low-profile growth of copper interconnection structure and reduce transmission loss to effectively ensure the integrity of high-frequency signal transmission.
\end{abstract}

Keywords: high-frequency signal, copper interconnection structure, electrochemical growth, organic additives, insertion loss

doi: $10.1360 /$ SSC-2021-0156 